\title{
Timing Acquisition Dispensing with Searching for UWB Communications
}

\author{
Zhiyuan Ren and Tiejun Lv, Member, IEEE \\ School of Information and Communication Engineering, \\ Beijing University of Posts and Telecommunications, Beijing, China 100876 \\ Email: \{rensimo, lvtiejun\}@gmail.com
}

\begin{abstract}
One of the biggest challenges in ultra-wideband (UWB) communications is the accurate and rapid timing acquisition for the receiver. This paper presents a timing acquisition scheme dispensing with searching for UWB communications. Without training sequence involved, timing offset parameter is estimated by exploiting a stream of alternating orthogonal pulses, and frame-level synchronization can be achieved without channel information at a low sampling rate of once per symbol. Without any searching procedure, the proposed algorithm not only considerably reduces synchronization time, but also improves synchronization performance relative to the existing alternatives in terms of normalized mean square error (NMSE) and bit error rate (BER), which is confirmed by simulations.
\end{abstract}

Index Terms-Ultra-Wideband impulse radio, Synchronization, Timing Acquisition, Dispensing with searching.

\section{INTRODUCTION}

Since the spectral mask was released by the Federal Communications Commission (FCC) in February 2002, UltraWideband impulse radio (UWB-IR) has received increasing attention especially in the area of short-range indoor wireless communications. Interest in UWB is motivated by several features including: high data rates along with low transmission power; high user capacity with time hopping $(\mathrm{TH})$ codes; potential spectrum compatibility with legacy services and robustness against multipath [1].

UWB-IR wireless communication system transmits a stream of ultra-short pulses (on the order of sub-nanoseconds) at very low power density. To obtain adequate signal energy for reliable detection, each information-bearing symbol is conveyed by a number of frames with one pulse per frame. The frame duration is much larger than the pulse duration, resulting in a very low duty cycle UWB transmission [1]. In such lowduty-cycle systems, accurate and rapid timing acquisition (i.e. synchronization) constitutes a major challenge in realizing the unique advantages of UWB impulse radio [2].

A number of algorithms have been developed to address this challenge [3]-[6]. However, each of these approaches inevitably requires a searching procedure, in which an objective function constructed from the received UWB signals is computed at dozens of candidate time shifts and the

This research is financially supported by Specialized Research Fund for the Doctoral Program of Higher Education (SRFDP) grant No. 20070013018 and Program for New Century Excellent Talents in University (NCET) under Grant No. NCET-06-0091. unique candidate time shift corresponding to the maximum (or minimum) of the objective function is regarded as synchronization parameter. Generally speaking, searching space will be enlarged as accuracy of synchronization is increased, thus leading to a lengthened synchronization time. Too long synchronization time will affect symbol detection.

Recently a class of synchronization schemes without searching procedure has been put forward [7]-[9]. The data-aided algorithm [7] partitions the synchronization parameter into symbol-level, frame-level and pulse-level timing offsets. Although the frame-level timing offset estimator is expressed in a closed form, it is actually based on a searching procedure due to its dependence on the searching-based estimator of the symbol-level offset. The data-aided synchronization scheme [8] is performed through energy comparison. However, it is tailored for the case that an information symbol consists of only one frame, thus not enabling multiple accesses. Moreover, the timing offset is obtained relying on channel information, but in practice, channel information is commonly unknown to the receiver at synchronization stage. Data-aided (DA) and non data-aided (NDA) schemes without searching procedure are introduced by [9]. A fixed preamble pattern of three symbols are transmitted before training sequence in DA mode and before information-bearing symbols in NDA mode, respectively. The authors take it for grant that the receiver begin synchronization at the instant, located within the first symbol of the fixed preamble pattern. However, this is infeasible in the "cold start-up" scenario [3] such as in wireless ad hoc and sensor networks, where the receiver does not know when the transmitter begins to send data. It is noteworthy that in the NDA mode of [9], the timing offset is derived in the absence of noise, whereas in the presence of noise the NDA scheme is not applicable unless signal to noise ratio (SNR) is high enough.

In this paper, a frame-level synchronization algorithm dispensing with searching is proposed. This algorithm is composed of two steps. In the first step, a stream of alternating orthogonal pulses is adopted to assist in estimating the tail energy as well as the head energy of the received symbollong waveform. In the second step, synchronization parameter is calculated on the basis of the estimates of the tail energy and the head energy. Unlike the existing searching-based synchronization algorithms [3]-[7], our approach does not 
need for a time-consuming searching procedure, therefore considerably shortening the synchronization time. Different from the training based algorithm [8], our timing offset estimator is derived without knowledge on channel. Compared with the schemes in [9], the proposed algorithm remains operational for "cold start-up" scenario and does not need any training pattern or sequence, thus not sacrificing bandwidth and transmission efficiency. Simulation confirms that the proposed scheme offers considerable performance improvements relative to the NDA mode in [9], and that performance degradation of the proposed algorithm in the presence of pulse distortion is quite limited.

The rest of this paper is organized as follows. Section II outlines our system model. Section III explains the proposed synchronization algorithm in detail. Simulations and comparisons are provided in section IV. At the end of this paper, summarizing remark is given.

\section{SYSTEM MODELING AND PRELIMINARIES}

In UWB-IR communications, every information symbol is conveyed by $N_{f}$ repeated pulses, with one pulse per frame of duration $T_{f}$. The resulting transmitted symbol-long waveform is described by

$$
p_{T}(t)=\sum_{j=0}^{N_{f}-1} p\left(t-j T_{f}-c_{j} T_{c}\right),
$$

where $p(t)$ is the normalized ultra-short pulse of duration $T_{p}$ at sub-nanosecond scale (a.k.a. monocycle). The sequence $\left\{c_{j}\right\}$ represents the user's pseudo-random time-hopping $(\mathrm{TH})$ code to enable multiple access and its elements are integers in the range $0 \leq c_{j} \leq N_{h}-1$, satisfying $T_{f} \geq\left(N_{h}-1\right) T_{c}+T_{p}$. $T_{c}$ is the duration of an addressable time bin. As a result, the symbol duration is $T_{s}:=N_{f} T_{f}$. Binary antipodal pulse amplitude modulation (PAM) is considered in this paper. The UWB modulated signal at the transmitter is then expressed as

$$
s(t)=\sqrt{\varepsilon} \sum_{i=0}^{+\infty} b(i) p_{T}\left(t-i T_{s}\right),
$$

where $\varepsilon$ is the transmitted energy per pulse, $b(i) \in\{ \pm 1\}$ is the information-bearing symbol with equal probability. The multipath channel is assumed to be quasi-stationary, i.e. the amplitude $\left\{\alpha_{l}\right\}$ and the delay $\left\{\tau_{l}\right\}$ of each path is invariant over one transmission burst. The impulse response of a $L-$ path channel can be denoted as:

$$
h(t)=\sum_{l=0}^{L-1} \alpha_{l} \delta\left(t-\tau_{l}\right)
$$

Without loss of generality, we assume $\tau_{0}<\tau_{1}<\ldots<\tau_{L-1}$ and isolate the propagation delay $\tau_{0}$ from multipath dispersion as $\tau_{l, 0}:=\tau_{l}-\tau_{0}, \forall l \in[0, L-1]$. With the receiver frontend acting as an ideal bandpass filter with ultra-wide bandwidth $B$, the received signal is then given by

$$
r(t)=\sqrt{\varepsilon} \sum_{i=0}^{+\infty} b(i) \sum_{l=0}^{L-1} \alpha_{l} p_{T}\left(t-i T_{s}-\tau_{0}-\tau_{l, 0}\right)+n(t)
$$

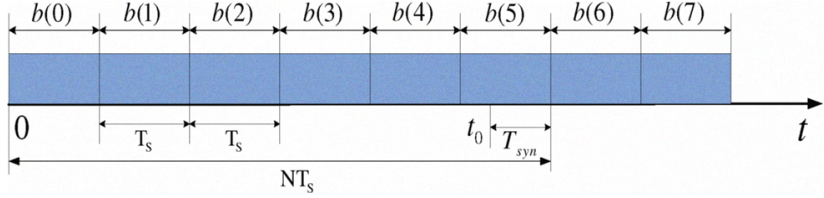

Fig. 1. The received signal with synchronization parameter

where $n(t)$ is the bandpass-filtered mixture of thermal noise and multiple-access interference (MAI), which is modeled as bandpass-filtered zero-mean additive Gaussian noise (AGN) with power spectral density (PSD) $N_{0} / 2$. Evidently, by selecting $T_{f} \geq \tau_{L-1,0}+T_{p}+\left(N_{h}-1\right) T_{c}$, the inter-frame interference (IFI) and the inter-symbol interference (ISI) can be avoided effectively [5]. For notational simplicity, we introduce the received symbol-long waveform

$$
\begin{aligned}
p_{R}(t) & :=\sum_{l=0}^{L-1} \alpha_{l} p_{T}\left(t-\tau_{l, 0}\right) \\
& =\sum_{j=0}^{N_{f}-1} g\left(t-j T_{f}-c_{j} T_{c}\right),
\end{aligned}
$$

where $g(t):=\sum_{l=0}^{L-1} \alpha_{l} p\left(t-\tau_{l, 0}\right)$ is the channel response of monocycle. It follows that the received waveform in (4) can be rewritten as

$$
r(t)=\sqrt{\varepsilon} \sum_{i=0}^{+\infty} b(i) p_{R}\left(t-i T_{s}-\tau_{0}\right)+n(t) .
$$

Although the received signal starts at $\tau_{0}$, the receiver knows neither the transmission starting time, nor the propagation delay $\tau_{0}$. Upon detecting the energy/amplitude change in the arriving signals, the receiver initiates synchronization at $t_{0}\left(t_{0}>\tau_{0}\right)$. Due to $\tau_{0}$ only serving as reference, without loss of generality, we set $\tau_{0}=0$. Therefore, the observation signal at the receiver can be formulated as

$$
\begin{aligned}
x(t) & :=r\left(t+t_{0}\right) \\
& =\sqrt{\varepsilon} \sum_{i=0}^{+\infty} b(i) p_{R}\left(t-i T_{s}+t_{0}\right)+n\left(t+t_{0}\right),
\end{aligned}
$$

where $t \in[0,+\infty)$ and $t_{0}$ can be expressed as an integer multiple of symbol duration $T_{s}$ minus a residue, yielding

$$
t_{0}=N T_{s}-T_{s y n}
$$

In (8), $N=\left\lceil t_{0} / T_{s}\right\rceil$ and $T_{s y n}=\left(N T_{s}-t_{0}\right) \in\left[0, T_{s}\right)$ is synchronization parameter to be estimated, as shown in Fig. 1 ( $\lceil\cdot\rceil$ stands for integer ceiling operation). Substituting (8) into (7) and letting $n^{\prime}(t)=n\left(t+t_{0}\right)$ give

$$
x(t)=\sqrt{\varepsilon} \sum_{i=0}^{+\infty} b(i) p_{R}\left(t-(i-N) T_{s}-T_{s y n}\right)+n^{\prime}(t) .
$$




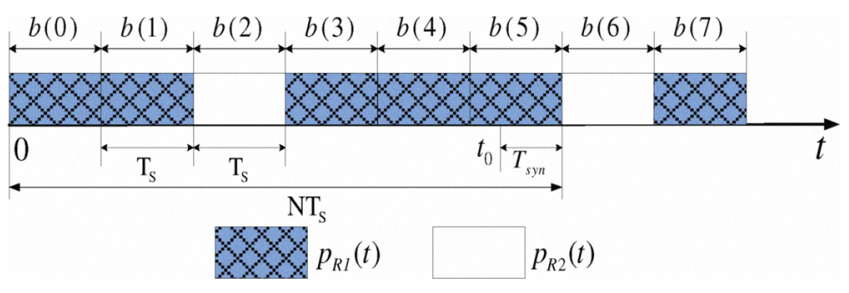

Fig. 2. The received signal when orthogonal pulses are employed

\section{SYNCHRONIZATION ALGORITHM}

Our novel synchronization scheme adopts two orthogonal consecutive-order Hermite pulses $p_{1}(t)$ and $p_{2}(t)$ with the same duration $T_{p}$, proposed in [10]. During each symbol, $p_{1}(t)$ or $p_{2}(t)$ is repeatedly transmitted in $N_{f}$ frames to convey one bit information. The transmitted monocycle is changed according to judiciously designed repeated pattern $\left[p_{1}(t), p_{1}(t), p_{2}(t), p_{1}(t)\right]$. The transmitted and received symbol-long waveforms are denoted by $p_{T 1}(t)$ and $p_{R 1}(t)$ when $p_{1}(t)$ is transmitted, and by $p_{T 2}(t)$ and $p_{R 2}(t)$ otherwise. According to (9), the observation signal becomes

$$
\begin{array}{r}
x(t)=\sqrt{\varepsilon} \sum_{i=0}^{+\infty} b(i) p_{R j(i)}\left(t-(i-N) T_{s}-T_{s y n}\right)+n^{\prime}(t) \\
j(i) \in\{1,2\}, t \in[0,+\infty),
\end{array}
$$

where the index $j(i) \in\{1,2\}$ indicates which one between $p_{1}(t)$ and $p_{2}(t)$ is received within the $i-t h$ symbol, as shown in Fig. 2.

Subsequently, we first estimate the tail energy as well as the head energy of the received symbol-long waveform, and then calculate the synchronization parameter on the basis of the estimates of the tail energy and the head energy.

\section{A. Estimating tail energy and head energy}

Firstly, inter-symbol correlation of the observation signal is performed to generate the symbol-rate samples:

$$
Z_{k}^{0}:=\int_{0}^{T_{s}} x\left(t+4 k T_{s}\right) x\left(t+(4 k+1) T_{s}\right) d t, k \in[0, K-1]
$$

where $x\left(t+k T_{s}\right)$ for $t \in\left[0, T_{s}\right)$ is the $k-t h$ segment waveform of the observation signal. According to (10), $x\left(t+k T_{s}\right)$ for $t \in\left[0, T_{s}\right)$ is then expressed by

$$
\begin{aligned}
x\left(t+k T_{s}\right) & =\sqrt{\varepsilon} \sum_{i=0}^{+\infty} b(i) p_{R j(i)}\left(t-(i-N-k) T_{s}-T_{s y n}\right) \\
& +n^{\prime}\left(t+k T_{s}\right), t \in\left[0, T_{s}\right), j(i) \in\{1,2\} .
\end{aligned}
$$

Since the received symbol-long waveform $p_{R j(i)}(t), j(i) \in$ $\{1,2\}$ has finite nonzero support $\left[0, T_{s}\right),(12)$ is zero except a finite number of $i$ values. For any given $k$ and $t_{0}$, it is not difficult to find that

$$
i=N+k-q,
$$

where $q$ is 0 or 1 . Substituting (13) into (12) yields

$$
\begin{aligned}
x\left(t+k T_{s}\right) & =\sqrt{\varepsilon} \sum_{q=0}^{1} b(N+k-q) p_{R j}\left(t+q T_{s}-T_{s y n}\right) \\
& +n^{\prime}\left(t+k T_{s}\right), t \in\left[0, T_{s}\right), j \in\{1,2\} .
\end{aligned}
$$

Therefore, when $N \bmod 4=1(\bmod$ represents modulo operation), $Z_{k}^{0}$ in (11) can be written as

$$
\begin{aligned}
Z_{k}^{0}=\varepsilon \cdot b(N-1+4 k) b(N+4 k) \cdot \int_{T_{s}-T_{s y n}}^{T_{s}} p_{R 1}^{2}(t) d t \\
+\varepsilon \cdot b(N+4 k) b(N+4 k+1) \cdot \\
\quad \int_{0}^{T_{s}} p_{R 1}\left(t-T_{s y n}\right) p_{R 2}\left(t-T_{s y n}\right) d t+\zeta(k),
\end{aligned}
$$

where $\zeta(k)$ stands for the Gaussian noise term with zero mean and variance $\sigma_{\zeta}^{2}$ [4]. We define $\varepsilon_{T i}\left(T_{s y n}\right):=$ $\varepsilon \int_{T_{s}-T_{s y n}}^{T_{s}} p_{R i}^{2}(t) d t, i i^{\zeta} \in\{1,2\}$ as the tail energy of the received symbol-long waveform $p_{R i}(t), \varepsilon_{H i}\left(T_{s y n}\right):=$ $\varepsilon \int_{0}^{T_{s}-T_{s y n}} p_{R i}^{2}(t) d t$ as the head energy of $p_{R i}(t)$ and $\varepsilon_{R i}:=$ $\varepsilon \int_{0}^{T_{s}} p_{R i}^{2}(t) d t$ as the total energy of $p_{R i}(t)$. We now make the following assumption [11], [12]

$$
\int_{-\infty}^{+\infty} p_{i}\left(t-\tau_{l, 0}\right) p_{j}\left(t-\tau_{k, 0}\right) d t \approx 0, i, j \in\{1,2\},
$$

for $l \neq k$. Invoking assumption (16) results in $\varepsilon_{T 1}\left(T_{s y n}\right)=$ $\varepsilon_{T 2}\left(T_{s y n}\right), \varepsilon_{H 1}\left(T_{s y n}\right)=\varepsilon_{H 2}\left(T_{s y n}\right)$, and $\varepsilon_{R 1}=\varepsilon_{R 2}$. Thus the above three types of energy are denoted by $\varepsilon_{T}\left(T_{\text {syn }}\right), \varepsilon_{H}\left(T_{\text {syn }}\right)$ and $\varepsilon_{R}$. The orthogonality between $p_{1}(t)$ and $p_{2}(t)$ combined with assumption (16) gives rise to $\int_{0}^{T_{s}} p_{R i}\left(t-T_{s y n}\right) p_{R j}\left(t-T_{s y n}\right) d t=0, i, j \in\{1,2\}$ for $i \neq j$. Therefore, $Z_{k}^{0}$ can be simplified to

$$
Z_{k}^{0}=b(N-1+4 k) b(N+4 k) \varepsilon_{T}\left(T_{s y n}\right)+\zeta(k) .
$$

Then, other three symbol-rate samples are obtained by performing correlations as follows:

$$
\begin{array}{r}
Z_{k}^{1}:=\int_{0}^{T_{s}} x\left(t+(4 k+1) T_{s}\right) x\left(t+(4 k+2) T_{s}\right) d t, \\
Z_{k}^{2}:=\int_{0}^{T_{s}} x\left(t+(4 k+2) T_{s}\right) x\left(t+(4 k+3) T_{s}\right) d t, \\
Z_{k}^{3}:=\int_{0}^{T_{s}} x\left(t+(4 k+3) T_{s}\right) x\left(t+(4 k+4) T_{s}\right) d t, \\
k \in[0, K-1] .
\end{array}
$$

When $N \bmod 4=1$, mimicking the steps to compute $Z_{k}^{0}, Z_{k}^{1}$, $Z_{k}^{2}$ and $Z_{k}^{3}$ can be expressed as

$$
\begin{aligned}
& Z_{k}^{1}=\zeta(k), \\
& Z_{k}^{2}=b(N+4 k+2) b(N+4 k+3) \varepsilon_{H}\left(T_{\text {syn }}\right)+\zeta(k), \\
& Z_{k}^{3}=b(N+4 k+2) b(N+4 k+3) \varepsilon_{T}\left(T_{\text {syn }}\right) \\
& \quad+b(N+4 k+3) b(N+4 k+4) \varepsilon_{H}\left(T_{\text {syn }}\right)+\zeta(k) .
\end{aligned}
$$

When $N \bmod 4=1$, taking the mean square of $Z_{k}^{i}, i \in[0,3]$ yields:

$$
\left\{\begin{array}{l}
E\left\{\left[Z_{k}^{0}\right]^{2}\right\}=\left[\varepsilon_{T}\left(T_{\text {syn }}\right)\right]^{2}+\sigma_{\zeta}^{2} \\
E\left\{\left[Z_{k}^{1}\right]^{2}\right\}=\sigma_{\zeta}^{2} \\
E\left\{\left[Z_{k}^{2}\right]^{2}\right\}=\left[\varepsilon_{H}\left(T_{\text {syn }}\right)\right]^{2}+\sigma_{\zeta}^{2} \\
E\left\{\left[Z_{k}^{3}\right]^{2}\right\}=\left[\varepsilon_{T}\left(T_{\text {syn }}\right)\right]^{2}+\left[\varepsilon_{H}\left(T_{\text {syn }}\right)\right]^{2}+\sigma_{\zeta}^{2} .
\end{array}\right.
$$


When $N \bmod 4=2,3,0$, the values of $E\left\{\left[Z_{k}^{i}\right]^{2}\right\}, i \in[0,3]$ is a circularly shifted version of (18). To identify the value of $N \bmod 4$, we first employ the following decision rule to determine whether $N \bmod 4=1 \operatorname{or} 3$ or $N \bmod 4=0$ or 2 :

$$
\left\{\begin{array}{r}
\left|E\left\{\left[Z_{k}^{0}\right]^{2}\right\}-E\left\{\left[Z_{k}^{2}\right]^{2}\right\}\right| \leq\left|E\left\{\left[Z_{k}^{1}\right]^{2}\right\}-E\left\{\left[Z_{k}^{3}\right]^{2}\right\}\right|, \\
N \bmod 4=1 \text { or } 3 \\
\left|E\left\{\left[Z_{k}^{0}\right]^{2}\right\}-E\left\{\left[Z_{k}^{2}\right]^{2}\right\}\right| \geq\left|E\left\{\left[Z_{k}^{1}\right]^{2}\right\}-E\left\{\left[Z_{k}^{3}\right]^{2}\right\}\right|, \\
N \bmod 4=0 \text { or } 2
\end{array}\right.
$$

where the equality holds $\forall k$ if and only if (iff) $T_{\text {syn }}=0$. If $N \bmod 4=1$ or 3 , the following decision rule is used to identify whether $N \bmod 4=1$ or $N \bmod 4=3$ :

$$
\left\{\begin{array}{r}
E\left\{\left[Z_{k}^{0}\right]^{2}\right\}-E\left\{\left[Z_{k}^{1}\right]^{2}\right\}>E\left\{\left[Z_{k}^{2}\right]^{2}\right\}-E\left\{\left[Z_{k}^{3}\right]^{2}\right\} \\
N \bmod 4=1 \\
E\left\{\left[Z_{k}^{0}\right]^{2}\right\}-E\left\{\left[Z_{k}^{1}\right]^{2}\right\}<E\left\{\left[Z_{k}^{2}\right]^{2}\right\}-E\left\{\left[Z_{k}^{3}\right]^{2}\right\} \\
N \bmod 4=3
\end{array}\right.
$$

If $N \bmod 4=2 o r 0$, the following decision rule is used to identify whether $N \bmod 4=2$ or $N \bmod 4=0$ :

$$
\left\{\begin{array}{l}
E\left\{\left[Z_{k}^{0}\right]^{2}\right\}<E\left\{\left[Z_{k}^{2}\right]^{2}\right\}, N \bmod 4=2, \\
E\left\{\left[Z_{k}^{0}\right]^{2}\right\}>E\left\{\left[Z_{k}^{2}\right]^{2}\right\}, N \bmod 4=0 .
\end{array}\right.
$$

Having known $N \bmod 4=n, n \in\{0,1,2,3\}$, tail energy $\varepsilon_{T}\left(T_{s y n}\right)$ and head energy $\varepsilon_{H}\left(T_{s y n}\right)$ can be readily computed as

$$
\left\{\begin{array}{l}
\varepsilon_{T}\left(T_{\text {syn }}\right)=\sqrt{E\left\{\left[Z_{k}^{(m-1) \bmod 4}\right]^{2}\right\}-E\left\{\left[Z_{k}^{m}\right]^{2}\right\}}, \\
\varepsilon_{H}\left(T_{\text {syn }}\right)=\sqrt{E\left\{\left[Z_{k}^{(m+1) \bmod 4}\right]^{2}\right\}-E\left\{\left[Z_{k}^{m}\right]^{2}\right\}},
\end{array}\right.
$$

where $m=n$ when $n$ is odd, and $m=(n+2) \bmod 4$ when $n$ is even. As usual, the ensemble mean square of the symbolrate samples $E\left\{\left[Z_{k}^{i}\right]^{2}\right\}, i \in[0,3]$ will be replaced in practice by its sample mean estimator obtained from $K$ symbol-rate samples $X_{i}:=K^{-1} \sum_{k=0}^{K-1}\left[Z_{k}^{i}\right]^{2}$.

\section{B. Estimating synchronization parameter}

In light of the multi-frame structure within each symbol in the absence of IFI, $\varepsilon_{T}\left(T_{s y n}\right)$ and $\varepsilon_{H}\left(T_{s y n}\right)$ can be expressed as:

$$
\begin{aligned}
& \varepsilon_{T}\left(T_{s y n}\right)=m \varepsilon_{h}\left(T_{f}\right)-\varepsilon_{h}\left(\eta-c_{N_{f}-m} T_{c}\right), \\
& \varepsilon_{H}\left(T_{s y n}\right)=\left(N_{f}-m\right) \varepsilon_{h}\left(T_{f}\right)+\varepsilon_{h}\left(\eta-c_{N_{f}-m} T_{c}\right),
\end{aligned}
$$

where $\varepsilon_{h}(x):=\varepsilon \int_{0}^{x} g^{2}(t) d t$ is accumulated multipath energy function, $m:=\left\lceil T_{s y n} / T_{f}\right\rceil \in\left\{1,2 \cdots N_{f}\right\}$ is an unknown integer and $\eta:=m T_{f}-T_{\text {syn }} \in\left[0, T_{f}\right)$ is referred to as tracking error. Adding (23) to (24), $\varepsilon_{h}\left(T_{f}\right)$ is given by

$$
\varepsilon_{h}\left(T_{f}\right)=\frac{\varepsilon_{H}\left(T_{\text {syn }}\right)+\varepsilon_{T}\left(T_{\text {syn }}\right)}{N_{f}} .
$$

Then, dividing both sides of (23) by $\varepsilon_{h}\left(T_{f}\right)$ gives

$$
\frac{\varepsilon_{T}\left(T_{s y n}\right)}{\varepsilon_{h}\left(T_{f}\right)}=m-\frac{\varepsilon_{h}\left(\eta-c_{N_{f}-m} T_{c}\right)}{\varepsilon_{h}\left(T_{f}\right)}=m-\varepsilon^{\prime},
$$

where $\varepsilon^{\prime}=\varepsilon_{h}\left(\eta-c_{N_{f}-m} T_{c}\right) / \varepsilon_{h}\left(T_{f}\right) \in[0,1]$. When $\varepsilon_{T}\left(T_{s y n}\right) / \varepsilon_{h}\left(T_{f}\right)$ is an integer, it is obvious that $t_{0}$ is located at the starting point of a frame. Consequently, we can estimate the synchronization parameter $T_{s y n}$ as:

$$
\hat{T}_{s y n}=\frac{\varepsilon_{T}\left(T_{s y n}\right)}{\varepsilon_{h}\left(T_{f}\right)} T_{f} .
$$

When $\varepsilon_{T}\left(T_{s y n}\right) / \varepsilon_{h}\left(T_{f}\right)$ is not an integer, according to (26) $m$ and $\varepsilon^{\prime}$ can be obtained as $m=\left\lceil\varepsilon_{T}\left(T_{\text {syn }}\right) / \varepsilon_{h}\left(T_{f}\right)\right\rceil$ and $\varepsilon^{\prime}=\left\lceil\varepsilon_{T}\left(T_{s y n}\right) / \varepsilon_{h}\left(T_{f}\right)\right\rceil-\varepsilon_{T}\left(T_{s y n}\right) / \varepsilon_{h}\left(T_{f}\right)$. With the definition of tracking error $\eta=m T_{f}-T_{s y n}$ and $\varepsilon^{\prime}=\varepsilon_{h}\left(\eta-c_{N_{f}-m} T_{c}\right) / \varepsilon_{h}\left(T_{f}\right)$, we can express $T_{s y n}$ and $\varepsilon_{h}\left(\eta-c_{N_{f}-m} T_{c}\right)$ as:

$$
\begin{aligned}
& T_{s y n}=m T_{f}-\eta=\left\lceil\varepsilon_{T}\left(T_{s y n}\right) / \varepsilon_{h}\left(T_{f}\right)\right\rceil T_{f}-\eta, \\
& \varepsilon_{h}\left(\eta-c_{N_{f}-m} T_{c}\right)=\varepsilon^{\prime} \times \varepsilon_{h}\left(T_{f}\right) .
\end{aligned}
$$

Notice that $\eta$ can not be recovered through the equation (28), for the accumulated multipath energy function $\varepsilon_{h}(x)$ is unknown at synchronization stage. As $t_{0}$ is randomly located within a symbol, it is reasonable to assume that $\eta$ obeys uniform distribution over $\left[0, T_{f}\right)$. Hence, in minimum mean square error (MMSE) sense, the optimal estimate of $\eta$ is $\hat{\eta}=T_{f} / 2$. As a result, the frame-level estimate of the synchronization parameter $T_{s y n}$ is given by

$$
\hat{T}_{\text {syn }}=\left\lceil\varepsilon_{T}\left(T_{\text {syn }}\right) / \varepsilon_{h}\left(T_{f}\right)\right\rceil T_{f}-T_{f} / 2 .
$$

After channel estimation at the receiver, the estimated accumulated multipath energy function $\hat{\varepsilon}_{h}(x)$ can be employed to estimate $\eta$ with more accuracy through the equation (28), which will in return makes channel estimation more accurate. Although our synchronization algorithms are developed when the channel information is unknown, these findings suggest an effectively iterative estimation process between synchronization parameter estimation and channel estimation, which finally improves bit error rate (BER) performance of the receiver.

Summarizing, the proposed synchronization algorithm is carried out as follows:

Step1. Use $X_{i}=K^{-1} \sum_{k=0}^{K-1}\left[Z_{k}^{i}\right]^{2}, i \in[0,3]$ to replace $E\left\{\left[Z_{k}^{i}\right]^{2}\right\}$ respectively in (19), (20), (21) and (22).

Step2. If the equality holds in (19), $\hat{T}_{s y n}=0$. Otherwise, the value of $N \bmod 4$ is obtained based on the decision rules in (19), (20), and (21), and then $\hat{\varepsilon}_{T}\left(T_{\text {syn }}\right)$ and $\hat{\varepsilon}_{H}\left(T_{\text {syn }}\right)$ are acquired according to (22).

Step3. Replace $\varepsilon_{H}\left(T_{s y n}\right)$ and $\varepsilon_{T}\left(T_{s y n}\right)$ in (25) with $\hat{\varepsilon}_{T}\left(T_{s y n}\right)$ and $\hat{\varepsilon}_{H}\left(T_{s y n}\right)$ to yield an estimate of $\varepsilon_{h}\left(T_{f}\right)$, i.e., $\hat{\varepsilon}_{h}\left(T_{f}\right)=\left[\hat{\varepsilon}_{T}\left(T_{s y n}\right)+\hat{\varepsilon}_{H}\left(T_{\text {syn }}\right)\right] / N_{f}$.

Step4. If $\hat{\varepsilon}_{T}\left(T_{s y n}\right) / \hat{\varepsilon}_{h}\left(T_{f}\right)$ is an integer, the frame-level estimate of synchronization parameter $\hat{T}_{s y n}=\left[\hat{\varepsilon}_{T}\left(T_{s y n}\right) / \hat{\varepsilon}_{h}\left(T_{f}\right)\right] T_{f}$. Otherwise, $\hat{T}_{s y n}=$ $\left\lceil\hat{\varepsilon}_{T}\left(T_{s y n}\right) / \hat{\varepsilon}_{h}\left(T_{f}\right)\right\rceil T_{f}-T_{f} / 2$.

\section{SIMULATIONS AND COMPARISIONS}

In this section, simulations are carried out to validate the performance of the proposed synchronization algorithm and compare it with that of NDA scheme in [9]. In all test cases, the transmitted monocycle is chosen as the second derivative of a Gaussian function with unit energy and duration $T_{p}=1 n s$ 


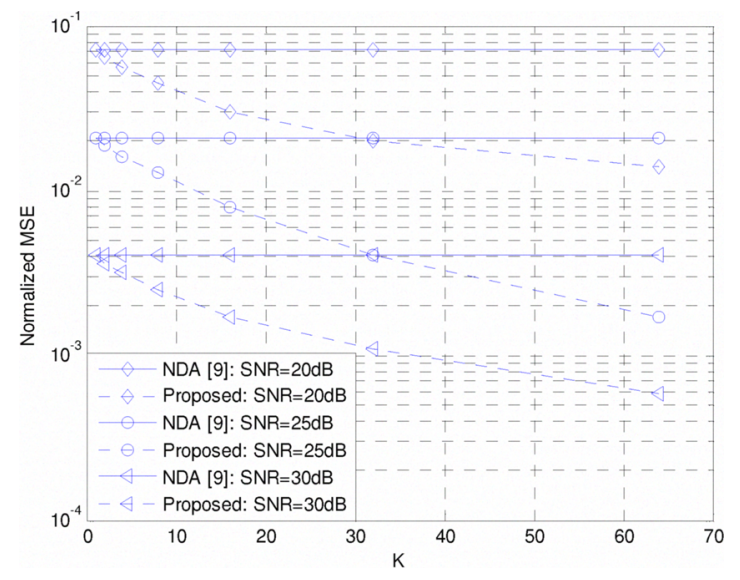

Fig. 3. MSE performance comparison with varying $\mathrm{K}$ at different SNR

for NDA scheme in [9]; while in the proposed algorithm we select the 3rd-order and 4th-order Hermite pulses with duration $T_{p}=1 n s$ as the alternatively transmitted monocycles. Each symbol consists of $N_{f}=13$ frames with frame duration $T_{f}=$ 40ns. For TH code, We set $N_{h}=10$ and $T_{c}=1 n s$. The fading channel is modeled as CM1 proposed by the IEEE 802.15 Working Group [13]. The diminishing tail of the power profile is truncated to make the maximum delay spread of the channel equal to $30 \mathrm{~ns}$. Without loss of generality, $t_{0}$ is randomly generated from a uniform distribution over $\left[0,4 T_{s}\right)$, and the observation interval of the received signal $T_{o b s}=$ $(4 K+1) T_{s}$ for $K=1,2,3 \cdots$.

We first compare performances between the proposed algorithm and NDA scheme in [9] in terms of NMSE, i.e., MSE normalized by $T_{s}^{2}$. Fig. 3 depicts NMSE of the proposed algorithm and that of NDA scheme in [9] with varying $K$ at different SNR. Here SNR is defined as $E_{b} / N_{0}$, where $E_{b}$ is the energy per symbol of the received signal. As shown in Fig. 3, at each SNR level, NMSE of the proposed algorithm reduces as $\mathrm{K}$ is increasing. On the contrary, NMSE of NDA scheme in [9] is almost invariant with $\mathrm{K}$ increasing, which results from the fact that the timing offset estimator in the NDA scheme of [9] is derived in the absence of noise and it is not consistent. Therefore, the proposed timing offset estimator is more effective than that of NDA scheme in [9]. Fig. 4 shows NMSE comparison with varying SNR at different $K$ values. The NMSE curves of the proposed algorithm with $K=32$ and 64 are below that of NDA in [9] with $K=64$. It reveals that the performance of the proposed algorithm is much superior to that of NDA scheme in [9].

In addition, we make BER performance comparison associated with the proposed algorithm and NDA scheme of [9] in Fig. 5. To isolate timing from channel estimation errors, the noise-free template is constructed with error-free channel for demodulation. As expected, with increased SNR or K, BER performance of the proposed algorithm improves monotonically. Even with $\mathrm{K}=32$, the proposed strategy provides far better BER performance than NDA scheme in [9] with $\mathrm{K}=64$.

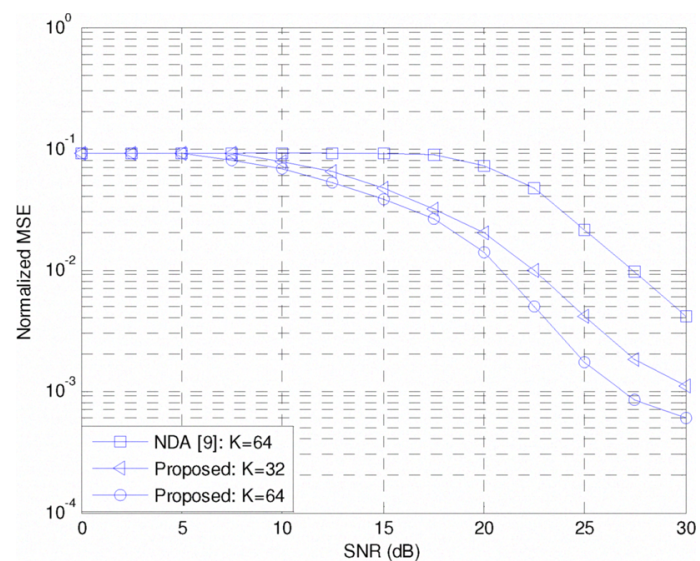

Fig. 4. MSE performance comparison with varying SNR at different $\mathrm{K}$

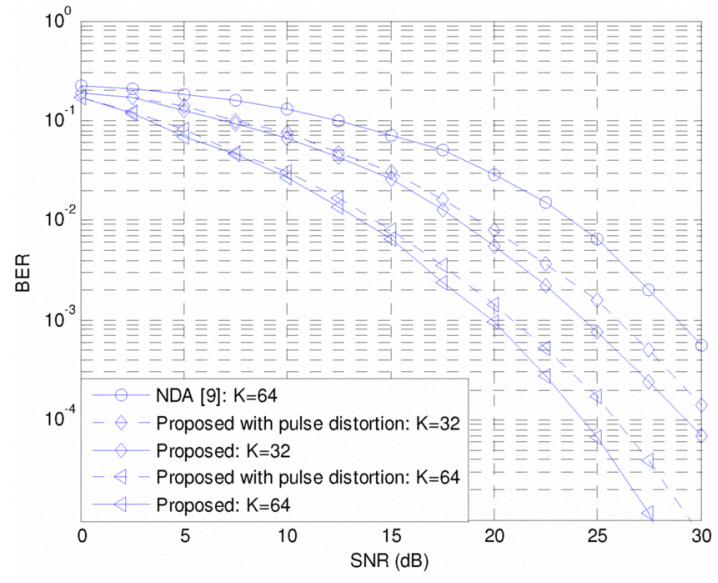

Fig. 5. BER performance comparison

Finally, we evaluate performance of the proposed algorithm in the presence of pulse distortion caused by transceiver's antennas [10] and propagation [14]. The effects of the transceiver's antennas are modeled as twice differential effects on the transmitted monocycle [10]. After twice differential, the transmitted 3rd-order and 4th-order Hermite monocycles become 5th-order and 6th-order ones, which are still orthogonal. We employ the generalized function [14, equation (2)] to model propagation-caused pulse distortion. In Fig. 5, dot lines represent BER performance of the proposed algorithm with pulse distortion. It is observed that BER performance degradation caused by pulse distortion is just within $2 \mathrm{~dB}$.

\section{CONCLUSIONS}

This paper introduces a synchronization algorithm dispensing with searching for UWB-IR wireless communication systems. Unlike searching-based synchronization algorithms, our approach does not require a time-consuming searching procedure, thus greatly shortening the synchronization time. Without any training pattern or sequence, the proposed algorithm can 
achieve frame-level synchronization at a low sampling rate of once per symbol. Compared with the NDA algorithm in [9], the proposed algorithm is operational for "cold start-up" scenario and provides considerable performance improvements.

In the future, we will focus on fine synchronization (tracking) algorithms in need of no searching process. Although the proposed synchronization algorithms are developed when the channel information is unknown, the estimation accuracy of the synchronization parameter will be improved significantly when the accumulated multipath energy function is known. Hence, our synchronization parameter estimation methods suggest an effectively iterative estimation process between synchronization parameter estimation and channel estimation, which finally improves BER performance of the receiver. This will be the direction of our future work.

\section{REFERENCES}

[1] M. Win and R. Scholtz, "Ultra-wide bandwidth time-hopping spreadspectrum impulse radio forwireless multiple-access communications," IEEE Transactions on communications, vol. 48, no. 4, pp. 679-689, 2000.

[2] Z. Tian and G. Giannakis, "BER sensitivity to mistiming in ultrawideband impulse radios-part II: fading channels," IEEE Transactions on Signal Processing, vol. 53, no. 5, pp. 1897-1907, 2005.

[3] L. Yang and G. Giannakis, "Blind UWB timing with a dirty template," in IEEE International Conference on Acoustics, Speech, and Signal Processing, 2004. Proceedings.(ICASSP'04), vol. 4, 2004.

[4] —-, "Timing ultra-wideband signals with dirty templates," IEEE Transactions on Communications, vol. 53, no. 11, pp. 1952-1963, 2005.

[5] C. Carbonelli and U. Mengali, "Synchronization algorithms for UWB signals," IEEE Transactions on Communications, vol. 54, no. 2, pp. 329-338, 2006.

[6] N. He and C. Tepedelenlioglu, "Joint Pulse and Symbol Level Acquisition of UWB Receivers," IEEE Transactions on Wireless Communications, vol. 7, no. 1, pp. 6-14, 2008.

[7] Z. Tian and G. Giannakis, "A GLRT approach to data-aided timing acquisition in UWB radios-Part I: Algorithms," IEEE Transactions on Wireless Communications, vol. 4, no. 6, pp. 2956-2967, 2005.

[8] D. Shin, Y. Cho, and D. Park, "A new synchronization scheme exploiting mean energy profile in UWB non-coherent receiver," in IEEE International Conference on Communications, 2006. ICC'06, vol. 12, 2006.

[9] J. Wang, L. Mai, Y. Peng, J. Han, and X. Zeng, "An Energy-Proportion Synchronization Method for IR-UWB Communications," in IEEE International Symposium on Circuits and Systems, 2007. ISCAS 2007, 2007, pp. 2578-2581.

[10] T. Montoya and G. Smith, "A study of pulse radiation from several broad-band loaded monopoles," IEEE Transactions on Antennas and Propagation, vol. 44, no. 8, pp. 1172-1182, 1996.

[11] C. Carbonelli and U. Mitra, "Clustered ML channel estimation for ultrawideband signals," IEEE Transactions on Wireless Communications, vol. 6 , no. 7, pp. 2412-2416, 2007.

[12] V. Lottici, A. D'Andrea, and U. Mengali, "Channel estimation for ultra-wideband communications," IEEE Journal on Selected Areas in Communications, vol. 20, no. 9, pp. 1638-1645, 2002.

[13] J. Foerster and I. R\&D, "Channel Modeling Sub-committee Report Final, IEEE P802. 15 Working Group for Wireless Personal Area Networks (WPANs)," IEEE P, vol. 15, no. 02.

[14] R. Qiu, C. Zhou, and Q. Liu, "Physics-based pulse distortion for ultrawideband signals," IEEE Transactions on Vehicular Technology, vol. 54, no. 5, pp. 1546-1555, 2005. 